\section{Protocol for Desensitization to Atezolizumab and Bevacizumab After Severe Anaphylaxis in the Treatment of Lung Adenocarcinoma}

Gonzalez-Diaz SN ${ }^{1}$, Villarreal-Gonzalez RV ${ }^{1}$, De LiraQuezada CE ${ }^{1}$, Rocha-Silva GK ${ }^{1}$, Oyervides-Juarez VM², VidalGutierrez $\mathrm{O}^{2}$

${ }^{\prime}$ Regional Center of Allergy and Clinical Immunology, University Hospital "Dr. Jose Eleuterio Gonzalez", Faculty of Medicine, Autonomous University of Nuevo León, Monterrey, Mexico ${ }^{2}$ Oncology Department, University Hospital "Dr. Jose Eleuterio Gonzalez", Faculty of Medicine, Autonomous University of Nuevo León, Monterrey, Mexico

J Investig Allergol Clin Immunol 2021; Vol. 31(3): 265-267 doi: $10.18176 /$ jiaci.0637

Key words: Adenocarcinoma. Anaphylaxis. Atezolizumab. Bevacizumab. Desensitization.

Palabras clave: Adenocarcinoma. Anafilaxia. Atezolizumab. Bevacizumab. Desensibilización.

Lung cancer is the most common cancer in the world, accounting for $19 \%$ of all cancer-related deaths [1]. Monoclonal antibodies (mAbs) have transformed the treatment of multiple diseases, including lung cancer [2], by inducing humoral and cellular immune responses, although they have also induced subsequent hypersensitivity reactions (HSRs) and infusion reactions (IRs). These reactions range from mild cutaneous manifestations to life-threatening anaphylaxis with hypotension, oxygen desaturation, cardiovascular collapse, and death [3].

Antineoplastic agents are the third leading cause of fatal drug-induced anaphylaxis in the United States. Given that severe HSRs threaten first-line treatments and affect survival, proper management of this problem is essential [4].

A 64-year-old man with no history of allergy was receiving treatment in 2017 for bladder cancer, which completely resolved with platinum salts. In March 2020, he was diagnosed with poorly differentiated lung adenocarcinoma with metastases to the adrenal glands and mediastinum (TNM classification, T4N3M1). The oncologist prescribed atezolizumab (1200 mg), bevacizumab (600 mg), carboplatin (4 AUC), and paclitaxel $\left(180 \mathrm{mg} / \mathrm{m}^{2}\right)$ every 3 weeks for 4 cycles. No HSRs were recorded during the first cycle of treatment. Thirty minutes after starting the second cycle of atezolizumab, the patient developed chest pain, hypotension, diaphoresis, and dizziness. The infusion was suspended. The symptoms resolved with intravenous hydrocortisone and diphenhydramine. Two days later, the second cycle of bevacizumab was administered. After 20 minutes, the patient developed hypotension, diaphoresis, cyanosis, hypoxia, and syncope (anaphylaxis grade 3 ), which were successfully managed with $0.5 \mathrm{mg}$ of intramuscular epinephrine, corticosteroids, and antihistamines.

The results of skin prick testing at $60 \mathrm{mg} / \mathrm{mL}$ of atezolizumab and $25 \mathrm{mg} / \mathrm{mL}$ of bevacizumab were negative. 
The results of intradermal skin tests at $0.6 \mathrm{mg} / \mathrm{mL}$ and $0.25 \mathrm{mg} / \mathrm{mL}$ were positive $(10 \times 10 \mathrm{~mm}$ and $13 \times 11 \mathrm{~mm}$, respectively $)$ compared with the negative control (glycerinated solution, $3 \times 3 \mathrm{~mm}$ ). Given the severity of the symptoms and need for treatment, desensitization protocols were performed 24 hours apart. The patient was premedicated with intravenous chlorpheniramine $(10 \mathrm{mg})$ and methylprednisolone $(60 \mathrm{mg})$ to decrease the intensity of symptoms and ondansetron $(8 \mathrm{mg})$ 1 hour prior to the infusion of the mAbs.

We administered $1200 \mathrm{mg}$ of atezolizumab and $600 \mathrm{mg}$ of bevacizumab on separate days in a protocol comprising 4 bags and 16 steps (initial concentration, 1:1000 of the total dose) (Table), administered at an increasing rate and concentration every 15 minutes without HSRs. We also administered fluids with normal saline solution at $100 \mathrm{~mL} / \mathrm{h}$ throughout the first 15 steps with an increase to $250 \mathrm{~mL} / \mathrm{h}$ after step 16 . Given that the patient tolerated the protocol without HSRs, we decided to perform a protocol comprising 3 bags and 12 steps (initial concentration, 1:100 of the total dose) every 3 weeks, which was successfully tolerated for 2 cycles. This protocol will be carried out for 1 year by indication of the oncologist as the first line of treatment.

Atezolizumab is a humanized immunoglobulin ( $\mathrm{Ig}) \mathrm{G}$ class 1 antibody that binds to programmed death ligand 1 (PD-L1). It has been approved for bladder, breast, and lung cancer. In 2016, the United States Food and Drug Administration reported severe IRs in $1.3 \%-1.7 \%$ and HSRs in $\leq 1 \%$ of cases [2].

Bevacizumab is a humanized IgG1 mAb that inhibits vascular endothelial growth factor, which is essential for normal and tumor angiogenesis and is considered first-line therapy for non-small cell lung cancer. HSRs to bevacizumab have been reported in $0.3 \%-6.1 \%$ of cases and manifest as dyspnea, erythema, hypotension, oxygen desaturation, chest pain, and nausea/vomiting [2].

HSRs are classified according to the time of onset and the mechanism involved as immediate ( $<1$ hour) or late $(>1$ hour $)$ and allergic or nonallergic. Immediate reactions include IRs, IgE-mediated cytokine release syndrome, and HSRs. They are clinically indistinguishable, and mixed-type reactions can be observed [5].

IRs are characterized by signs or symptoms experienced during the infusion of pharmacological or biological agents or any event that occurs on the first day of administration of the drug [6]. Reactions to mAbs appear most frequently from 10 minutes to 4 hours after starting administration. IRs are very similar to anaphylaxis, although they can be caused by multiple mechanisms, and anaphylaxis is an IgE-mediated reaction [7].

Brown et al [8] classify grade 1 anaphylaxis as involving skin symptoms, grade 2 systemic symptoms, and grade 3 severe symptoms such as syncope, incontinence, hypotension, or hypoxia. Standardized skin tests for mAbs have not been established. The recommendation is to wait 2 to 4 weeks after the reaction to avoid false negatives after anaphylaxis. Since positive skin tests indicate an IgE-mediated immune mechanism, the patient is a candidate for desensitization [9].

In vitro and in vivo models propose that in desensitization, mast cells and basophils can be induced in inhibitory pathways by small increasing doses of antigen, thus disabling signal transduction and mediator release. In severe anaphylaxis, delivering therapy starting at $1 / 1000$ of the target dose

Table. Four-Bag, 16-Step Protocol for Desensitization to Atezolizumab and Bevacizumab

\begin{tabular}{|c|c|c|c|c|c|c|c|c|}
\hline \multirow[t]{2}{*}{ Steps } & \multirow[t]{2}{*}{ Solution } & \multirow[t]{2}{*}{$\begin{array}{l}\text { Rate, } \\
\mathrm{mL} / \mathrm{h}\end{array}$} & \multirow[t]{2}{*}{$\begin{array}{c}\text { Time } \\
\min \end{array}$} & \multirow[t]{2}{*}{$\begin{array}{l}\text { Volume } \\
\text { per Step, } \\
\text { mL }\end{array}$} & $\begin{array}{c}\text { Dose } \\
\text { Administered, } \\
\mathrm{mg}\end{array}$ & $\begin{array}{c}\text { Cumulative } \\
\text { Dose, } \\
\text { mg }\end{array}$ & $\begin{array}{c}\text { Dose } \\
\text { Administered, } \\
\mathrm{mg}\end{array}$ & $\begin{array}{c}\text { Cumulative } \\
\text { Dose, } \\
\text { mg }\end{array}$ \\
\hline & & & & & \multicolumn{2}{|c|}{ Atezolizumab protocol } & \multicolumn{2}{|c|}{ Bevacizumab protocol } \\
\hline 1 & 1 & 2.5 & 15 & 0.625 & 0.003 & 0.003 & 0.0015 & 0.0015 \\
\hline 2 & 1 & 5 & 15 & 1.25 & 0.006 & 0.009 & 0.003 & 0.0045 \\
\hline 3 & 1 & 10 & 15 & 2.5 & 0.012 & 0.021 & 0.006 & 0.0105 \\
\hline 4 & 1 & 20 & 15 & 5 & 0.024 & 0.045 & 0.012 & 0.0225 \\
\hline 5 & 2 & 2.5 & 15 & 0.625 & 0.03 & 0.075 & 0.015 & 0.038 \\
\hline 6 & 2 & 5 & 15 & 1.25 & 0.06 & 0.135 & 0.03 & 0.068 \\
\hline 7 & 2 & 10 & 15 & 2.5 & 0.12 & 0.255 & 0.06 & 0.128 \\
\hline 8 & 2 & 20 & 15 & 5 & 0.24 & 0.5 & 0.12 & 0.248 \\
\hline 9 & 3 & 5 & 15 & 1.25 & 0.6 & 1.1 & 0.3 & 0.55 \\
\hline 10 & 3 & 10 & 15 & 2.5 & 1.2 & 2.3 & 0.6 & 1.15 \\
\hline 11 & 3 & 20 & 15 & 5 & 2.4 & 4.7 & 1.2 & 2.35 \\
\hline 12 & 3 & 40. & 15 & 10 & 4.8 & 9.5 & 2.4 & 4.75 \\
\hline 13 & 4 & 10 & 15 & 2.5 & 11.9 & 21.4 & 5.95 & 10.7 \\
\hline 14 & 4 & 20 & 15 & 5 & 23.8 & 45.2 & 11.9 & 22.6 \\
\hline 15 & 4 & 40 & 15 & 10 & 47.6 & 92.8 & 23.8 & 46.4 \\
\hline 16 & 4 & 80 & 174.4 & 232.5 & 1107.2 & 1200 & 553.6 & 600 \\
\hline
\end{tabular}


with a sufficient interval to avoid mast cell degranulation inhibits the acute release of $\beta$-hexosaminidase and prevents the generation of arachidonic acid and products such as leukotrienes and prostaglandins, as well as the late generation of inflammatory cytokines. Furthermore, entry of calcium is prevented, and the polymerization of actin is altered, thus stabilizing the intracellular granules in an antigen-specific manner and inducing temporary tolerance that protects from anaphylaxis [3].

The safest option for HSRs to a drug is to avoid it, although in patients with malignancies, switching to a second-line agent negatively affects quality of life and life expectancy. Patients with type 1 and cytokine-release reactions to $\mathrm{mAbs}$ are thought to be candidates for desensitization [10].

In terms of safety and efficacy, desensitization is highly effective and enables patients to receive first-line therapy with better clinical results and fewer complications, thus reducing mortality, morbidity, and expense in medical care $[11,12]$.

\section{Funding}

The authors declare that no funding was received for the present study.

\section{Conflicts of Interest}

The authors declare that they have no conflicts of interest.

\section{References}

1. Cheng TY, Cramb SM, Baade PD, Youlden DR, Nwogu C, Reid ME. The International Epidemiology of Lung Cancer: Latest Trends, Disparities, and Tumor Characteristics. J Thorac Oncol. 2016 Oct;11(10):1653-71.

2. Gülsen A, Wedi B, Jappe U. Hypersensitivity reactions to biologics (part I): allergy as an important differential diagnosis in complex immune-derived adverse events. Allergo J Int. 2020:1-29.

3. Castells M. Drug Hypersensitivity and Anaphylaxis in Cancer and Chronic Inflammatory Diseases: The Role of Desensitizations. Front Immunol. 2017;8:1472.

4. Madrigal-Burgaleta R, Bernal-Rubio L, Berges-Gimeno MP, Carpio-Escalona LV, Gehlhaar P, Alvarez-Cuesta E. A Large Single-Hospital Experience Using Drug Provocation Testing and Rapid Drug Desensitization in Hypersensitivity to Antineoplastic and Biological Agents. J Allergy Clin Immunol Pract. 2019;7(2):618-32.

5. Akarsu A, Soyer O, Sekerel BE. Hypersensitivity Reactions to Biologicals: from Bench to Bedside. Curr Treat Options Allergy. 2020;7(1):71-83.

6. Kang SP, Saif MW. Infusion-relate and hypersensitivity reactions of monoclonal antibodies used to treat colorectal cancer - identification, prevention, and management. J Support Oncol. 2007;5(9):451-7.

7. Cáceres MC, Guerrero-Martín J, Pérez-Civantos D, PalomoLópez P, Delgado-Mingorance Jl, Durán-Gómez N. The importance of early identification of infusion-related reactions to monoclonal antibodies. Ther Clin Risk Manag. 2019;15:965-77.
8. Brown SGA. Clinical features and severity grading of anaphylaxis. J Allergy Clin Immunol. 2004;114(2):371-6.

9. Hong DIC. Desensitization for Allergic Reactions to Chemotherapy. Yonsei Med J. 2019;60(2):119-25.

10. Isabwe GAC, Garcia Neuer M, de Las Vecillas Sanchez L, Lynch DM, Marquis K, Castells M. Hypersensitivity reactions to therapeutic monoclonal antibodies: Phenotypes and endotypes. J Allergy Clin Immunol. 2018;142(1):159-70.e2.

11. Berges-Gimeno MP, Carpio-Escalona LV, Longo-Muñoz $F$, Bernal-Rubio L, Lopez-Gonzalez P, Gehlhaar P, et al. Does Rapid Drug Desensitization to chemotherapy affect survival outcomes? J Investig Allergol Clin Immunol. 2020;30(4):1-29.

12. de Las Vecillas Sánchez L, Alenazy LA, Garcia-Neuer M, Castells MC. Drug Hypersensitivity and Desensitizations: Mechanisms and New Approaches. Int J Mol Sci. 2017;18(6):1316.

Manuscript received June 17, 2020; accepted for publication August 4, 2020.

Rosalaura Virginia Villarreal-Gonzalez E-mail: rosalauravillarrealg@gmail.com 